\title{
Valence band effective mass of non-c-plane nitride heterostructures
}

\author{
AUTHOR(S):
}

Kojima, K.; Funato, M.; Kawakami, Y.; Noda, S.

\section{CITATION:}

Kojima, K....[et al]. Valence band effective mass of non-c-plane nitride heterostructures. JOURNAL OF APPLIED PHYSICS 2010, 107(12): 123105.

\section{ISSUE DATE:}

2010-06

URL:

http://hdl.handle.net/2433/147193

\section{RIGHT:}

Copyright 2010 American Institute of Physics. This article may be downloaded for personal use only. Any other use requires prior permission of the author and the American Institute of Physics. The following article appeared in JOURNAL OF APPLIED PHYSICS107, 123105 (2010) and may be found at 


\title{
Valence band effective mass of non-c-plane nitride heterostructures
}

\author{
K. Kojima, ${ }^{\text {a) }}$ M. Funato, Y. Kawakami, and S. Noda \\ Department of Electronic Science and Engineering, Kyoto University, Kyoto 615-8510, Japan
}

(Received 6 April 2010; accepted 11 May 2010; published online 16 June 2010)

\begin{abstract}
Valence band (VB) effective masses of nonpolar and semipolar nitride heterostructures were studied using $\boldsymbol{k} \cdot \boldsymbol{p}$ perturbation theory, with a particular focus on band mixing. When the absolute value of the energy separation of the topmost two VBs $(|\Delta E|)$ is sufficiently larger than the spin-orbit interaction $\left(\Delta_{\mathrm{so}}\right)$, the mixing between these two VBs was negligible. This enabled us to calculate an analytical expression for VB mass expression. Under this condition, the effective mass of the top VB was reduced less than $10 \%$ along a certain direction, and less than $25 \%$ for an in-plane ( $x^{\prime} y^{\prime}$-plane) average, compared to the $c$-plane case. When $\Delta E$ was comparable with $\Delta_{\text {so }}$, however, the VB structure became isotropic due to band mixing. Consequently, the VB band structure approached that of the $c$-plane and optical polarization was suppressed. On the other hand, VB mass along the growth direction $\left(z^{\prime}\right)$ was always heavy, similar to that of the $c$-plane. However, the topmost two VBs had slightly different mass values along $z^{\prime}$, which affected radiative properties of non-c-plane nitride quantum wells (less than $2 \mathrm{~nm}$ wide) through the difference in quantum confinement strengths. (C) 2010 American Institute of Physics. [doi:10.1063/1.3448578]
\end{abstract}

\section{INTRODUCTION}

Radiative properties of nitride semiconductors on non- $c$-plane semipolar substrates and facets have been intensively investigated in recent years. Such extraordinary crystal orientation reduces the quantum-confined Stark effect (QCSE). ${ }^{1,2}$ The reported experimental results suggest potential application in non-c-plane light emitting diodes and laser diodes (LDs) as low-threshold and long-wavelength emitters. ${ }^{3-5}$

From the point of view of basic science, optical polarization is one of unique properties on the non- $c$-plane crystal orientation, and it has been investigated both theoretically and experimentally. ${ }^{6-11}$ Although the reduction in the valence band (VB) effective mass has been predicted much earlier, ${ }^{12,13}$ there have been no systematic discussions of this topic until now. In this article, we investigate the VB effective mass of nonpolar and semipolar nitride heterostructures theoretically, including InGaN/GaN and AlGaN/AlN, by analyzing VB electronic structures.

\section{THEORY}

VB structures can be computed numerically by employing $\boldsymbol{k} \cdot \boldsymbol{p}$ perturbation theory. ${ }^{14,15}$ The spatial rotation matrix has been applied for the $6 \times 6 \mathrm{VB}$ Hamiltonian to deal with the non-c-plane crystal orientation. ${ }^{12,13}$ The VB Hamiltonian of Wurtzite crystals is given by the following: ${ }^{16,17}$

\footnotetext{
${ }^{a)}$ Electronic mail: kkojima@qoe.kuee.kyoto-u.ac.jp.
}

$$
\boldsymbol{H}=\left(\begin{array}{cccccc}
F & -K^{*} & -H^{*} & & & \\
-K & G & H & & & \Delta \\
-H & H^{*} & \Lambda & & \Delta & \\
& & & F & -K & H \\
& & \Delta & -K^{*} & G & -H^{*} \\
& \Delta & & H^{*} & -H & \Lambda
\end{array}\right),
$$

where

$$
\begin{aligned}
& F=\Delta_{\mathrm{cr}}+\Delta_{\mathrm{so}} / 3+\Lambda+\Theta, \\
& G=\Delta_{\mathrm{cr}}-\Delta_{\mathrm{so}} / 3+\Lambda+\Theta, \\
& \Lambda=\frac{\hbar^{2}}{2 m_{0}}\left\{A_{1} k_{z}^{2}+A_{2}\left(k_{x}^{2}+k_{y}^{2}\right)\right\}+D_{1} \varepsilon_{z z}+D_{2}\left(\varepsilon_{x x}+\varepsilon_{y y}\right), \\
& \Theta=\frac{\hbar^{2}}{2 m_{0}}\left\{A_{3} k_{z}^{2}+A_{4}\left(k_{x}^{2}+k_{y}^{2}\right)\right\}+D_{3} \varepsilon_{z z}+D_{4}\left(\varepsilon_{x x}+\varepsilon_{y y}\right), \\
& K=\frac{\hbar^{2}}{2 m_{0}} A_{5}\left(k_{x}+\mathrm{i} k_{y}\right)^{2}+D_{5}\left(\varepsilon_{x x}-\varepsilon_{y y}+2 \mathrm{i} \varepsilon_{x y}\right), \\
& H=\frac{\hbar^{2}}{2 m_{0}} A_{6} k_{z}\left(k_{x}+\mathrm{i} k_{y}\right)+D_{6}\left(\varepsilon_{x z}+\mathrm{i} \varepsilon_{y z}\right), \\
& \Delta=\sqrt{2} \Delta_{\mathrm{so}} / 3 .
\end{aligned}
$$

Here $A$ and $D$ are the VB parameters and deformation potentials, respectively, $\varepsilon$ is the strain, and $\Delta_{\mathrm{cr}}$ and $\Delta_{\mathrm{so}}$ are the energies of the crystal-field splitting and the spin-orbit interaction, respectively. In addition, the wave numbers $\boldsymbol{k}_{i}$ ( $i$ $=x, y, z)$, where the $x y$-plane and the $z$-axis correspond to the $c$-plane and $c$-axis, respectively, and $\boldsymbol{k}_{i^{\prime}}$ in the $x^{\prime} y^{\prime} z^{\prime}$ system of coordinate are connected by a spatial rotation matrix as follows: 


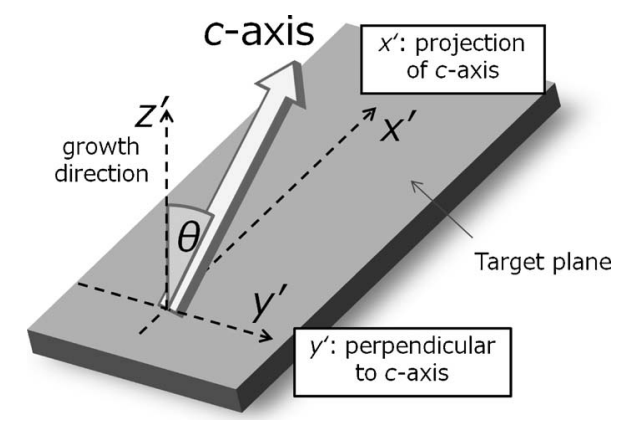

FIG. 1. Definition of the angle $\theta$, and the axes $x^{\prime}, y^{\prime}$, and $z^{\prime}$.

$$
k_{i^{\prime}}=\mathbf{R} k_{i},
$$

where

$$
\boldsymbol{R}=\left(\begin{array}{ccc}
\cos \phi \cos \theta & \sin \phi \cos \theta & -\sin \theta \\
-\sin \phi & \cos \phi & 0 \\
\cos \phi \sin \theta & \sin \phi \sin \theta & \cos \theta
\end{array}\right) .
$$

For simplicity, in-plane rotation $\phi$ is set to be 0 in our discussion. The crystal angle $\theta$ and the $x^{\prime}-, y^{\prime}-$, and $z^{\prime}$-axes are defined as shown in Fig. 1.

\section{ANALYTICAL SOLUTIONS FOR MATERIAL SYSTEMS WITHOUT SIGNIFICANT BAND MIXING}

When the absolute value of the energy separation of the topmost two VBs $(|\Delta E|)$ was sufficiently larger than $\Delta_{\text {so }}$, the analytical expression of the effective masses can be extracted, as shown in this section. It has been reported earlier that if reducing QCSE is desired along the growth directions, having a crystal orientation with $\theta$ higher than $55^{\circ}$ is important ${ }^{1,2}$ for both $\mathrm{InGaN} / \mathrm{GaN}$ and AlGaN/AlN. For such crystal planes, the above-mentioned condition $\left(|\Delta E| \gg \Delta_{\text {so }}\right)$ is usually satisfied, as strain-related energies in Eq. (2) are much larger than $\sqrt{2} \Delta_{\mathrm{so}} / 3$. The resulting expressions can be useful for practical applications as well as fundamental studies.

Neglecting $\sqrt{2} \Delta_{\mathrm{so}} / 3$, the Hamiltonian can be split into two parts

$$
\mathbf{H} \approx\left(\begin{array}{cc}
\mathbf{H}^{\mathrm{U}} & \\
& \mathbf{H}^{\mathrm{L}}
\end{array}\right),
$$

where

$$
\begin{aligned}
\mathbf{H}^{\mathrm{U}} & =\left(\begin{array}{ccc}
F & -K^{*} & -H^{*} \\
-K & F & H \\
-H & H^{*} & \Lambda
\end{array}\right), \text { and } \mathbf{H}^{\mathrm{L}} \\
& =\left(\begin{array}{ccc}
F & -K & H \\
-K^{*} & F & -H^{*} \\
H^{*} & -H & \Lambda
\end{array}\right) .
\end{aligned}
$$

This procedure is identical to the one carried out in Ref. 18, where it was performed in the zone center. For the case of $\theta=90^{\circ}, \mathbf{H}^{\mathrm{U}}$, and $\mathbf{H}^{\mathrm{L}}$ can be simplified because of the shear strain components vanish
TABLE I. VB effective masses $\left(m_{j} / m_{0}, j=1,2\right.$, and 3$)$ of nonpolar nitride heterostructures with (a) $\theta=90^{\circ}$ and (b) $\theta=0^{\circ} . m_{0}$ is the mass of static free-electron. A VB with the eigenenergy $E_{j}$ has the effective mass $m_{j} . m_{\mathrm{HH}}$, $m_{\mathrm{LH}}$, and $m_{\mathrm{CH}}$ represent masses of the heavy hole, the light hole, and the crystal-field splitting hole defined in the $c$-plane, respectively.

\begin{tabular}{lcccc}
\hline \hline & \multicolumn{2}{c}{ In-plane mass } & & Vertical mass \\
\cline { 2 - 3 } (a) $\theta=90^{\circ}$ & $x^{\prime}$-direction & $y^{\prime}$-direction & & $z^{\prime}$-direction \\
\hline$m_{1} / m_{0}$ & $-\left(A_{1}+A_{3}\right)^{-1}$ & $-\left(A_{2}+A_{4}+A_{5}\right)^{-1}$ & & $-\left(A_{2}+A_{4}-A_{5}\right)^{-1}$ \\
$m_{2} / m_{0}$ & $-A_{1}^{-1}$ & $-A_{2}^{-1}$ & & $-A_{2}^{-1}$ \\
$m_{3} / m_{0}$ & $-\left(A_{1}+A_{3}\right)^{-1}$ & $-\left(A_{2}+A_{4}-A_{5}\right)^{-1}$ & & $-\left(A_{2}+A_{4}+A_{5}\right)^{-1}$
\end{tabular}

\begin{tabular}{lcc} 
(b) $\theta=0^{\circ}$ & In-plane mass $\left(m^{\perp}\right)$ & Vertical mass $\left(m^{\|}\right)$ \\
\hline$m_{\mathrm{HH}} / m_{0}$ & $-\left(A_{2}+A_{4}-A_{5}\right)^{-1}$ & $-\left(A_{1}+A_{3}\right)^{-1}$ \\
$m_{\mathrm{LH}} / m_{0}$ & $-\left(A_{2}+A_{4}+A_{5}\right)^{-1}$ & $-\left(A_{1}+A_{3}\right)^{-1}$ \\
$m_{\mathrm{CH}} / m_{0}$ & $-A_{2}^{-1}$ & $-A_{1}^{-1}$ \\
\hline
\end{tabular}

$$
\mathbf{H}^{\mathrm{U}}=\left(\begin{array}{ccc}
F & -K^{*} & \\
-K & F & \\
& & \Lambda
\end{array}\right) \text { and } \mathbf{H}^{\mathrm{L}}=\left(\begin{array}{ccc}
F & -K & \\
-K^{*} & F & \\
& & \Lambda
\end{array}\right) \text {. }
$$

We can now obtain the degenerate eigenenergies of Eq. (7), which correspond to the three individual VBs

$$
\begin{aligned}
& E_{1}=F+|K|, \\
& E_{2}=\Lambda, \\
& E_{3}=F-|K| .
\end{aligned}
$$

In the zone center, the order of the VB eigenenergies [Eq. (8)] depends on the system in question. For example, $E_{1}$ $>E_{2}>E_{3}$ for nonpolar $\operatorname{In}_{x} \mathrm{Ga}_{1-x} \mathrm{~N} / \mathrm{GaN}$, and $E_{2}>E_{1}>E_{3}$ for nonpolar $\mathrm{Al}_{x} \mathrm{Ga}_{1-x} \mathrm{~N} / \mathrm{AlN}$ for any In or $\mathrm{Al}$ content due to a difference in the crystal-field splitting energy. Furthermore, it is possible to change the energy order of $E_{1}$ and $E_{2}$ by changing the $\mathrm{Al}$ composition of InGaN/AlGaN. ${ }^{11,18}$ We have reported an optical polarization switching phenomenon in $(11 \overline{2} 2)$ InGaN/GaN quantum wells (QWs), where we found that optical polarization is dependent on In composition. ${ }^{7}$ In that case, the energy order of $E_{1}$ and $E_{2}$ was dominated by the In content of InGaN. In addition, the $E_{3} \mathrm{VB}$ is always far from the other two bands, and we do not discuss it hereafter.

Substituting Eq. (2) into Eq. (8), we find the analytical VB effective masses as coefficients of $\boldsymbol{k}_{i^{\prime}}^{2}$ in the procedure described in Ref. 15. The VB effective masses $\left(m_{j}, j=1,2\right.$, and 3 ) corresponding to the eigenenergies $E_{j}$ are summarized in Table I, (a). Nonpolar InGaN/GaN or AlGaN/AlN always satisfies $|\Delta E| \gg \Delta_{\text {so }}$, so that the analytical solutions obtained are always applicable. For semipolar InGaN/GaN, however, there is a limitation, because $|\Delta E|$ strongly depends on In content. $K$ and $H$, which determine band-mixing in Eq. (6), satisfy the condition $|K| \geq|H|$, if $\theta>55^{\circ}$. Thus, Table I, (a), is also a good approximation for semipolar InGaN/GaN with $\theta>55^{\circ}$, for example $(11 \overline{2} 2)$, as long as $|\Delta E| \gg \Delta_{\text {so }}$ is satisfied, where $\Delta_{\text {so }}$ is $16 \mathrm{meV}$ for $\mathrm{GaN}$ and $5 \mathrm{meV}$ for $\mathrm{InN}$. These results are discussed in detail below. 
TABLE II. VB effective mass components $\left(m_{j} / m_{0}, j=1,2,3\right)$ of nitride semiconductors. All the parameters are given in the units of $m_{0}$.

\begin{tabular}{lccccc}
\hline \hline & $-\left(A_{1}+A_{3}\right)^{-1}$ & $-\left(A_{2}+A_{4}+A_{5}\right)^{-1}$ & $-A_{1}^{-1}$ & $-A_{2}^{-1}$ & $-\left(A_{2}+A_{4}-A_{5}\right)^{-1}$ \\
\hline $\mathrm{InN}$ & 1.563 & 0.091 & 0.112 & 1.471 & 1.250 \\
$\mathrm{GaN}$ & 1.889 & 0.137 & 0.139 & 2.272 & 2.000 \\
$\mathrm{AlN}$ & 3.571 & 0.329 & 0.259 & 4.000 & 10.00 \\
\hline \hline
\end{tabular}

In nitride semiconductors such as $\mathrm{InN}, \mathrm{GaN}$, and $\mathrm{AlN}$ the values $-\left(A_{2}+A_{4}+A_{5}\right)^{-1}$ and $-A_{1}^{-1}$ are much smaller than $-\left(A_{1}+A_{3}\right)^{-1},-\left(A_{2}+A_{4}-A_{5}\right)^{-1}$, and $-A_{2}^{-1}$, as shown in Table II. These parameters also appear in the VB mass set (far from the zone center) for the $c$-plane, as shown in Table I, (b). ${ }^{16,17}$ We discovered the following three important points from analytical solutions in Table I, (a).

(1) VB masses are determined only by VB parameters $A$ when $\Delta_{\text {so }}$ is negligible and they are independent of $D$. This is similar to the scenario of "far from zone center" in Ref. 17.

(2) The in-plane $\left(x^{\prime}-\right.$ and $\left.y^{\prime}-\right)$ components of $m_{1}$ and $m_{2}$ in Table I, (a), differ: one of them is light, so that the mean in-plane VB mass is reduced compared to the $c$-plane case. This phenomenon can potentially lower threshold carrier density for LDs.

(3) The vertical $\left(z^{\prime}-\right)$ components of both $m_{1}$ and $m_{2}$ are heavy. We thus predict that the current injection properties are similar to those of the $c$-plane case. However, $z^{\prime}$-components of $m_{1}$ and $m_{2}$ are slightly different, thus $\Delta E$ is not constant as a function of $\mathrm{QW}$ width for non-c-planes. We will discuss this topic in detail below.

The analytical expressions in Table I, (a), thus, give a clear understanding of the effective VB mass.

\section{NUMERICAL ANALYSES}

We have also analyzed the VB structure numerically. ${ }^{10}$ The energy dependence of the effective mass along the $i^{\prime}$ direction of the three separate VBs has been calculated using the $E-k$ dispersions obtained as curvatures. ${ }^{19-21}$ The spinsplit bands were averaged and the epilayers were assumed to be coherently grown on GaN or AlN substrates, even for strained films or QWs.

The order of $E_{1}$ and $E_{2}$ of semipolar and nonpolar nitride heterostructures can be summarized as shown in Table III. As mentioned above, the conditions $E_{1}>E_{2}$ [regime (I)] for nonpolar $\operatorname{In}_{x} \mathrm{Ga}_{1-x} \mathrm{~N} / \mathrm{GaN}$, and $E_{2}>E_{1}$ [regime (III)] for nonpolar $\mathrm{Al}_{x} \mathrm{Ga}_{1-x} \mathrm{~N} / \mathrm{AlN}$ are satisfied. On the other hand, semipolar InGaN/GaN shows crossover between $E_{1}$ and $E_{2}$ at a certain In content, and therefore, has to be classified as regime (I), (II), where $E_{1} \sim E_{2}$, or (III), depending on the crystal angle and In content, as shown in Fig. 2. The solid line in Fig. 2 represents the In content where crossover between $E_{1}$ and $E_{2}$ occurs and, consequently, optical polarization is switched. One should keep in mind that this switching for In content is strongly dependent on the reliability of strain and deformation potential parameters, especially for $D_{5}$ and $D_{6}$, which appear in $K$ and $H$ of the VB Hamiltonian Eq. (2) as the coefficients of the anisotropic strain components. Here we chose these values from Ref. 7 to reproduce experimental trends, i.e., optical polarization switching. Other parameters were taken from Ref. 22.

Mixing of the topmost two VBs barely occurs in regimes (I) and (III), whereas band mixing is significant in regime (II). Therefore, the analytical expressions given in Sec. III can be used in regimes (I) and (III).

\section{A. In-plane mass $\left(m^{x^{\prime}}\right.$ and $\left.m^{y^{\prime}}\right)$ characters \\ 1. Regime I: $E_{1}>E_{2}$}

Figures 3(a)-3(c) show the $E-k$ relations for a nonpolar $\mathrm{In}_{0.1} \mathrm{Ga}_{0.9} \mathrm{~N} / \mathrm{GaN}$ strained film along $k_{x^{\prime}}, k_{y^{\prime}}$, and $k_{z^{\prime}}$. At the band edge in Fig. 3(b), the top VB mass along $y^{\prime}$ is greatly reduced compared to that of the $c$-plane. By contrast, the band edge mass of the second VB along $x^{\prime}$ is light. Moreover, the energy separation between the top and the second $(\Delta E)$ is larger than the typical value in the $c$-plane ( $\sim 9 \mathrm{meV}$ ). Hence,

(1) the dispersions are nearly parabolic due to reduced coupling of the two top VBs and effective masses are anisotropic.

(2) Large $\Delta E$ suppresses the hole population in the second VB. Thus recombination between the conduction band (CB) and the top VB dominates the radiative process compared to the $c$-plane case.

The first point makes the analytical mass expression given in Table I, (a), a robust approximation. Furthermore, we find that the ratio of VB masses $\left(m_{1}^{y^{\prime}} / m_{\mathrm{HH}}^{\perp}\right)$ is $7.3 \%$ along the $y^{\prime}$-axis, and the geometric mean in-plane mass ratio $\left(\sqrt{m_{1}^{x^{\prime}} m_{1}^{y^{\prime}}} / m_{\mathrm{HH}}^{\perp}\right)$ is $26.9 \%$ for the non-c-plane case. This is one of the advantages of the non- $c$-plane nitride heterostructures - reducing the in-plane density of states. We

TABLE III. The order of VB energies $E_{1}$ and $E_{2}$.

\begin{tabular}{lcc}
\hline \hline & Material & Optical polarization \\
\hline$E_{1}>E_{2}$ & Nonpolar InGaN/GaN, semipolar InGaN/GaN (low In) & $\sim+1$ (perpendicular to $c$-axis) \\
$E_{1} \sim E_{2}$ & Semipolar InGaN/GaN (see Fig. 5) & $\sim 0$ \\
$E_{1}<E_{2}$ & Semipolar InGaN/GaN (high In), nonpolar AlGaN/AlN & $\sim-1$ (along $c$-axis or its projection) \\
\hline
\end{tabular}




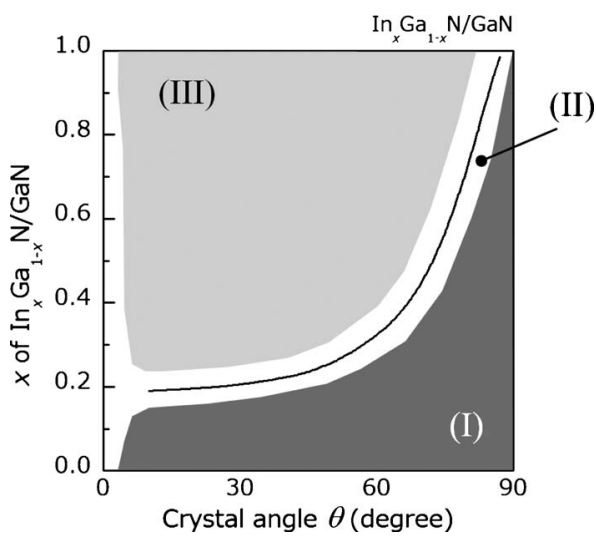

FIG. 2. Numerical results for the order of VB energy of InGaN/GaN as a function of crystal angle. The $c$-plane and its neighboring region belong to group (II). The nonpolar plane is placed in region (i) for any In content. The solid line represents the In content, in which $E_{1}=E_{2}$ under the assumption of $\Delta_{\mathrm{so}}=0$.

believe, moreover, that they also enhance in-plane mobility of VBs. The second point allows us to simulate the system by using a single parabolic dispersion for VB as well as CB. Large $\Delta E$ also improves the optical polarization linearity of radiation [see Eq. (6) of Ref. 10] and enhances the population inversion between $\mathrm{CB}$ and the top VB, which results in greater gain and lower threshold current for laser applications. Figures 3(d)-3(f) represent $E-k$ relations of (1122)
$\mathrm{In}_{0.1} \mathrm{Ga}_{0.9} \mathrm{~N} / \mathrm{GaN}$ strained film along $k_{x^{\prime}}, k_{y^{\prime}}$, and $k_{z^{\prime}}$. Compared to the nonpolar orientation case, $\Delta E$ is slightly smaller. We find, however, that the VB characteristics in nonpolar and $(11 \overline{2} 2)$ cases are nearly the same. This proves that the approximation in Eq. (5) is good even for semipolar planes.

\section{Regime II: $E_{1} \sim E_{2}$}

When $E_{1}$ and $E_{2}$ have similar values and $\Delta E$ becomes small, band-mixing through the spin-orbit interaction becomes significant in the zone center. In this regime (II), the basis of the two topmost VBs change from $\left|Y^{\prime}\right\rangle$ and $\left|X^{\prime}\right\rangle$, which appear in the regime (I), to $\left|X^{\prime} \pm \mathrm{i} Y^{\prime}\right\rangle$, and the analytical solution $E_{j}$ in Table I, (a), no longer gives the correct VB energies. We find the $E-k$ dispersion for (11/22) $\mathrm{In}_{0.28} \mathrm{Ga}_{0.72} \mathrm{~N} / \mathrm{GaN}$, in which In content is an experimentally obtained singularity for polarization switching, as shown in Figs. 4(a)-4(c). We also find that the in-plane $E-k$ relations and VB masses are nearly isotropic [Figs. 4(a) and 4(b)], and they are nearly identical to the in-plane $E-k$ dispersion of the $c$-plane, as shown in Fig. 4(d). Therefore, VB masses of both (11) 2$) \mathrm{In}_{0.28} \mathrm{Ga}_{0.72} \mathrm{~N} / \mathrm{GaN}$ and $c$-plane InGaN show similar trends. In this case, instead of analytical VB mass in Table I, (a), masses in $c$-plane-like $m_{\mathrm{HH}}$ and $m_{\mathrm{LH}}$ given in Table I, (b), are the correct representation. The VB energies become $E_{\mathrm{HH}}$ and $E_{\mathrm{LH}}$, which are defined in the $c$-plane. Incidentally, the (a) nonpolar, $x^{\prime}$
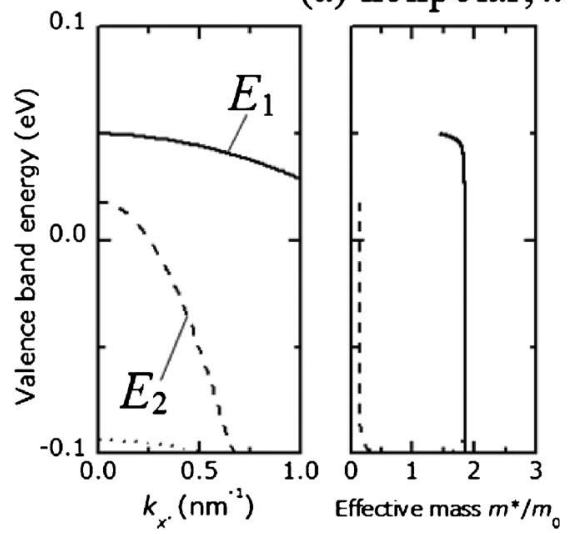

(d) semipolar, $x^{\prime}$
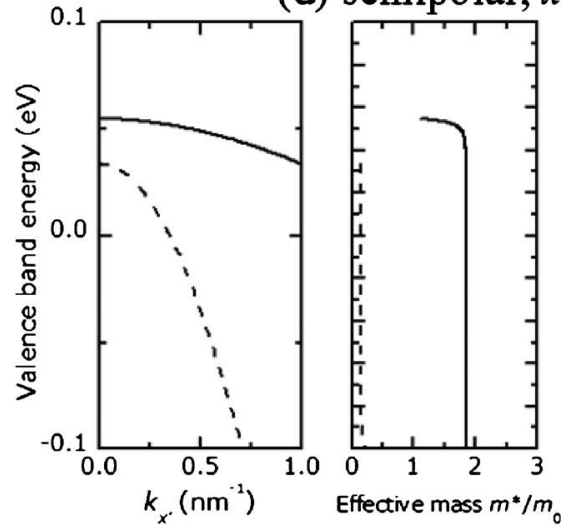

(b) nonpolar, $y^{\prime}$

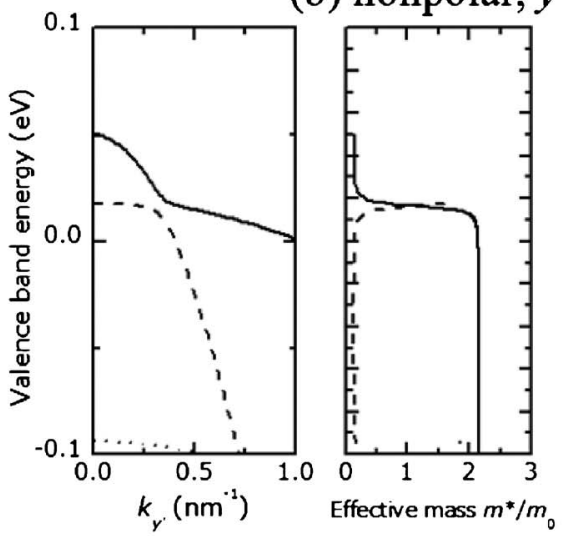

(e) semipolar, $y^{\prime}$
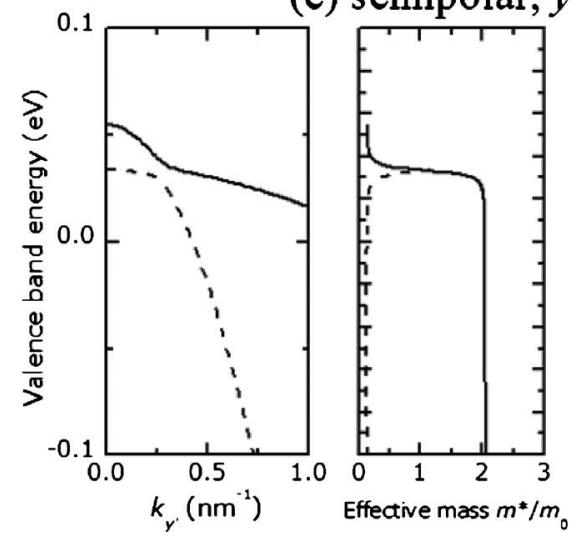

(c) nonpolar, $z^{\prime}$
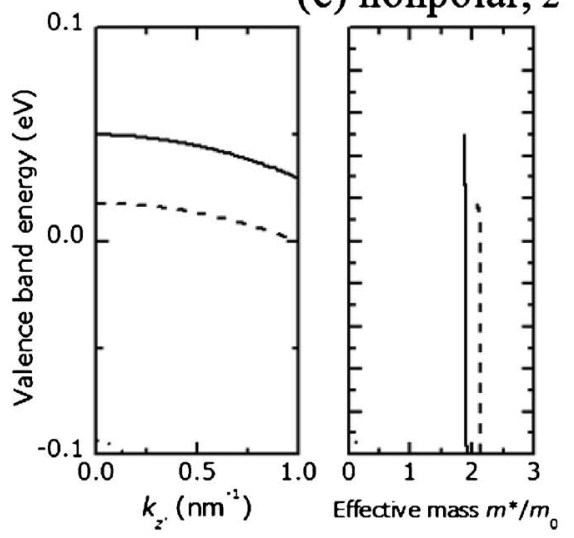

(f) semipolar, $z^{\prime}$
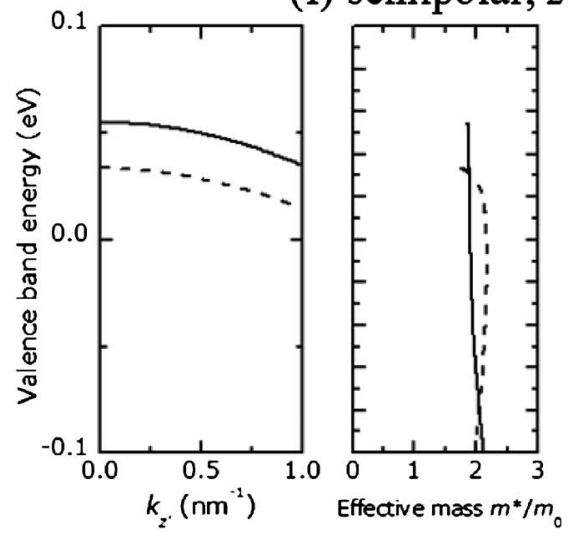

FIG. 3. VB energies and effective masses calculated for nonpolar $\operatorname{In}_{0.1} \mathrm{Ga}_{0.9} \mathrm{~N} / \mathrm{GaN}$, and $(11 \overline{2} 2) \mathrm{In}_{0.1} \mathrm{Ga}_{0.9} \mathrm{~N} / \mathrm{GaN}$ films. The solid and dashed lines represent the highest and second highest VBs, respectively. 
(a) semipolar, $x^{\prime}$
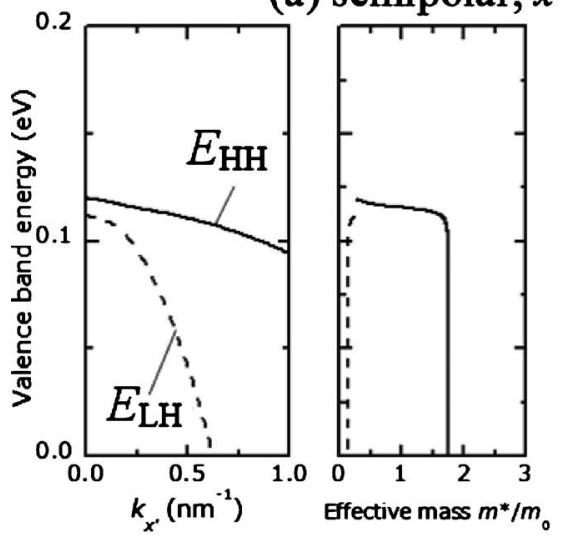

(b) semipolar, $y^{\prime}$

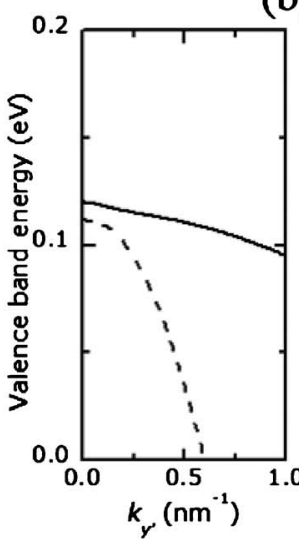

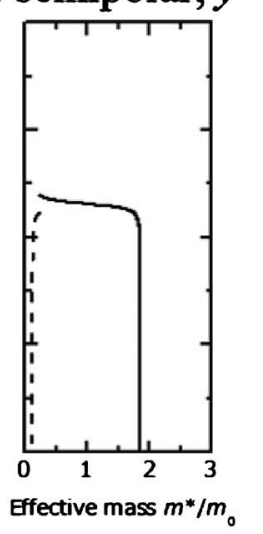

(c) semipolar, $z^{\prime}$
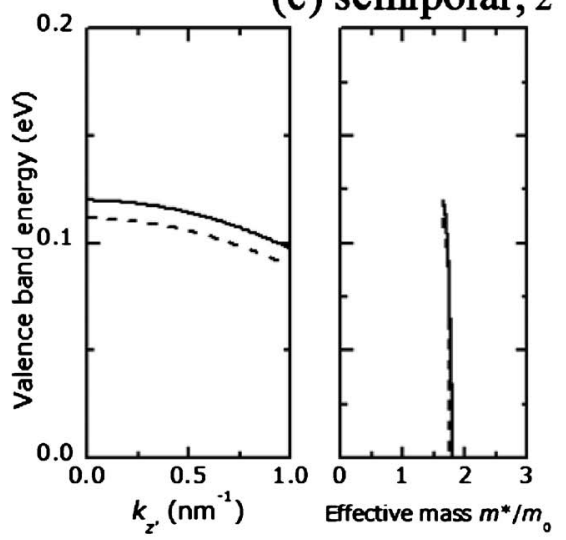

(d) c-plane, $\perp$
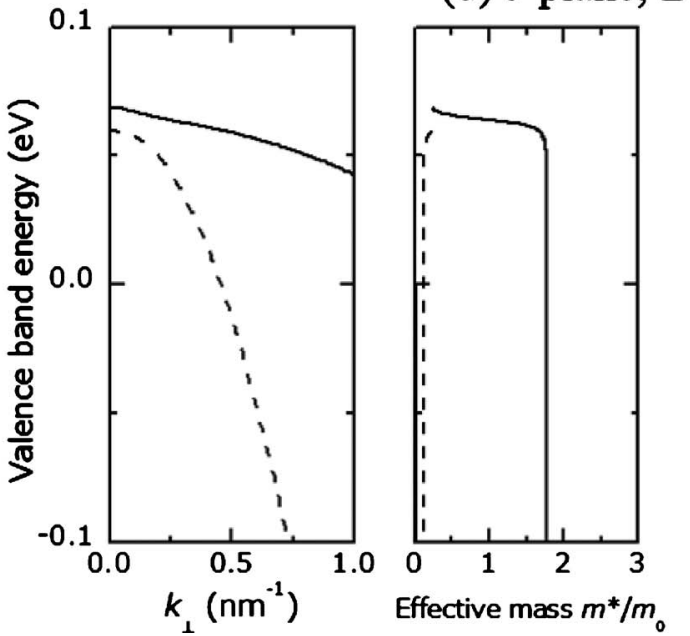

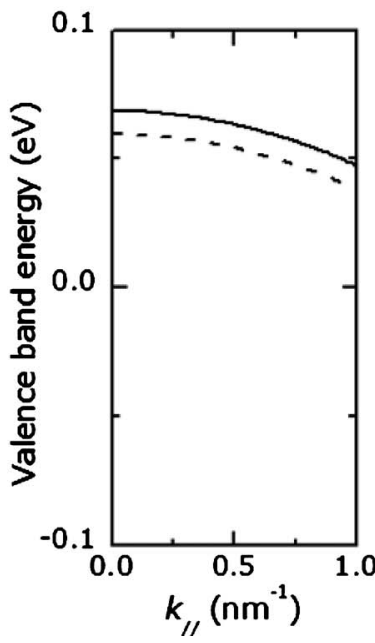

(e) c-plane, //

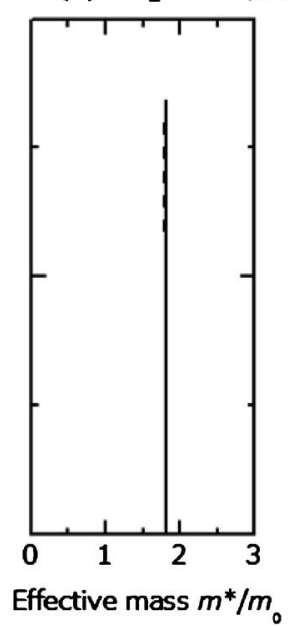

FIG. 4. VB energies and effective masses of a (11) 2$) \mathrm{In}_{0.28} \mathrm{Ga}_{0.72} \mathrm{~N} / \mathrm{GaN}$ film along (a) $k_{x^{\prime}}$, (b) $k_{y^{\prime}}$, and (c) $k_{z^{\prime}}$ and of a strained $c$-plane $\mathrm{In}_{0.2} \mathrm{Ga}_{0.8} \mathrm{~N} / \mathrm{GaN}$ film along (d) $k_{\perp}=k_{y^{\prime}}$ and (e) $k_{\|}=k_{z^{\prime}}$. The solid and dashed lines represent the highest and second highest VBs, respectively.

VB structure of $(11 \overline{2} 2) \operatorname{In}_{0.28} \mathrm{Ga}_{0.72} \mathrm{~N} / \mathrm{GaN}$ is very close to that of nonpolar $\mathrm{In}_{0.1} \mathrm{Ga}_{0.9} \mathrm{~N} / \mathrm{Al}_{0.23} \mathrm{Ga}_{0.77} \mathrm{~N}$ described in Ref. 18 .

\section{Regime III: $E_{1}<E_{2}$}

In contrast with the regime (I), the VB with the eigenenergy $E_{2}$ and eigenfunction $\left|X^{\prime}\right\rangle$ is the top $\mathrm{VB}$, as shown in Fig. 5. The reduced VB mass thus appears along $k_{x^{\prime}}$. Analytical solutions to Eq. (8) again work well in this case, because $|\Delta E| \geq \Delta_{\text {so }}$. As mentioned above, $E_{2}$ in the AlGaN/AlN structure is always larger than $E_{1}$, because $\Delta_{\text {cr }}$ is negative and its absolute value is quite large. Thus $E_{2}>E_{1}$ is rigorously maintained and appears to be independent from the uncertainty in the parameters as long as the Al content of $\mathrm{AlGaN}$ is below $5 \%$.

To summarize, we have found that the analytical VB mass solution is a good approximation for both regimes (I) and (III) due to large $|\Delta E|$. When $|\Delta E|$ becomes small, and band-mixing occurs in a significant way through $\Delta_{\text {so }}$, optical polarization decreases. In addition, band structures are strongly modified and become similar to the $c$-plane one in regime (II). The VB diagram of semipolar InGaN/GaN dependence on In content is summarized schematically in Fig. 6.

\section{B. Vertical mass $\left(m^{z^{\prime}}\right)$ characters}

The vertical mass $m_{z^{\prime}}$, which is the mass along the growth direction, chiefly affects the formation of a subband in QWs. Figures 7(a) and 7(b) indicate $|\Delta E|$ and the corre-

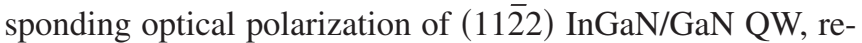
spectively, as a function of QW width $L_{\mathrm{w}}$. The energy difference $|\Delta E|$ in a $\mathrm{QW}$ is defined as the energy difference of the first two subbands: $|\Delta E|=\left|E_{1, n=1}-E_{2, n=1}\right|$, where $n$ is the subband index. $|\Delta E|$ and optical polarization are nearly constant for $L_{\mathrm{w}}>2 \mathrm{~nm}$, and their values are identical to those for $L_{\mathrm{w}}=\infty$ (strained film case). This is consistent with the reported experimental result. ${ }^{7}$ However, $|\Delta E|$ and optical polarization for $L_{\mathrm{w}}<2 \mathrm{~nm}$ are affected by $L_{\mathrm{w}}$ and depend on the In content. This is because (11) 2 ) InGaN/GaN belongs to regime (I), (II), and (III) with respect to In content, as discussed above. 
(a) semipolar, $x^{\prime}$
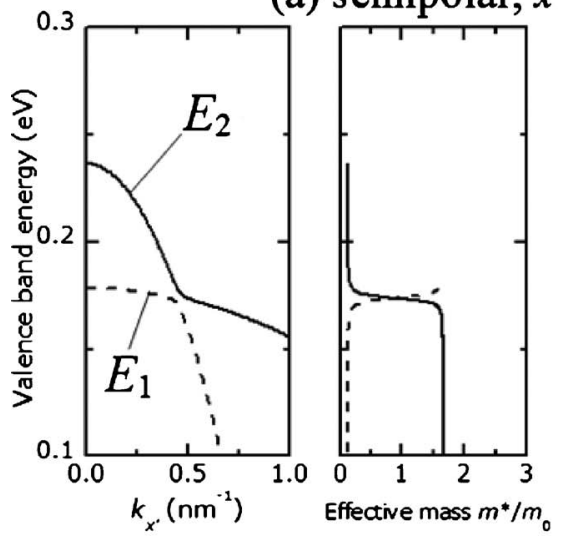

(d) nonpolar, $x^{\prime}$
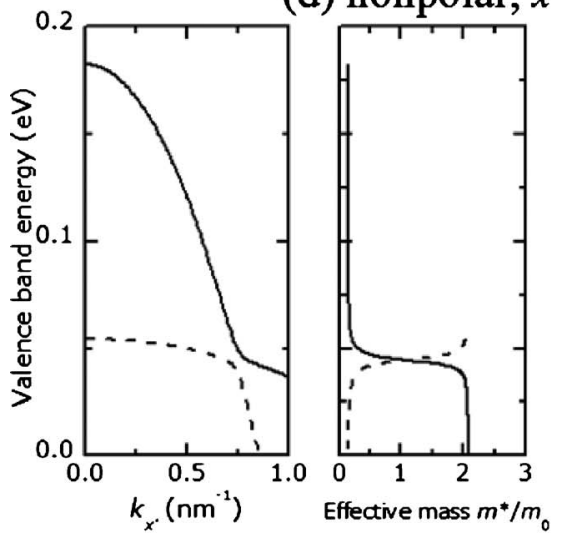

(b) semipolar, $y^{\prime}$
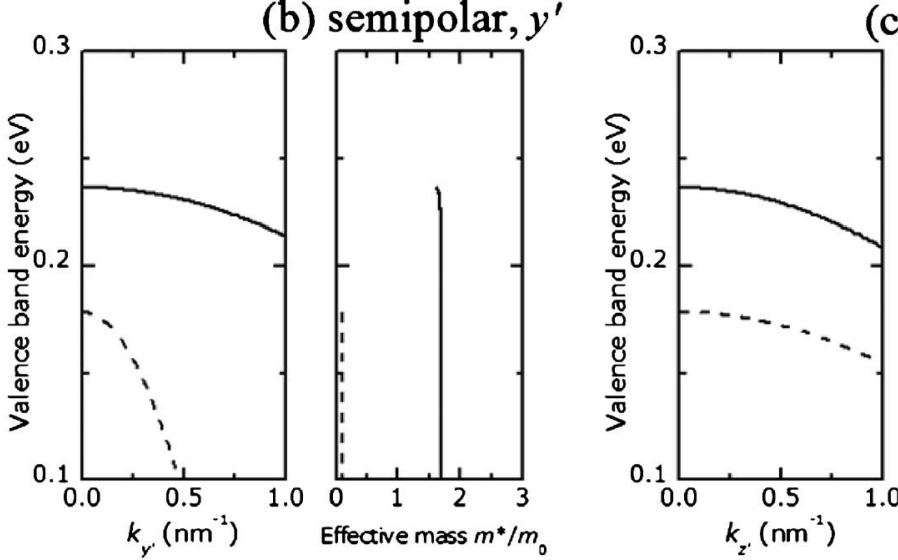

(c) semipolar, $z^{\prime}$

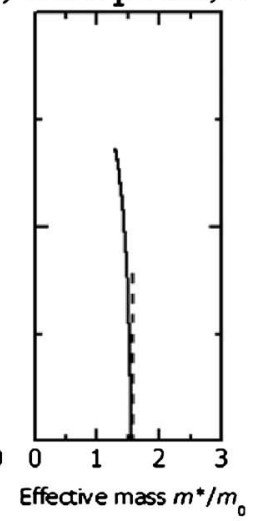

(e) nonpolar, $y^{\prime}$
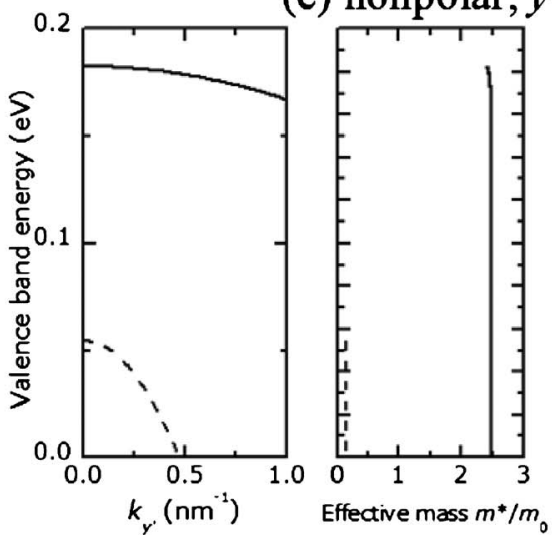

(f) nonpolar, $z^{\prime}$
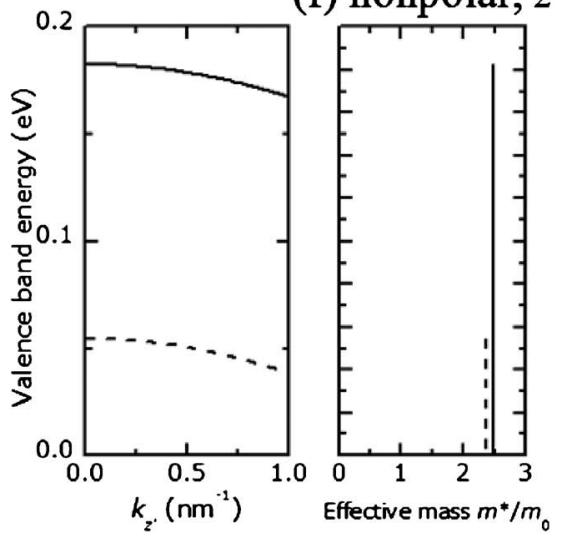

FIG. 5. VB energies and effective masses calculated for $(11 \overline{2} 2) \mathrm{In}_{0.5} \mathrm{Ga}_{0.5} \mathrm{~N} / \mathrm{GaN}$, and nonpolar $\mathrm{Al}_{0.2} \mathrm{Ga}_{0.8} \mathrm{~N} / \mathrm{AlN}$ films. The solid and dashed lines represent the highest and second highest VBs, respectively.

\section{Regime I: $E_{1}>E_{2}$}

In Fig. 7(a), lines with In content of 10 and $20 \%$ belong to regime (I). $|\Delta E|$ decreases with decreasing $L_{\mathrm{w}}$ for $L_{\mathrm{w}}$ $<2 \mathrm{~nm}$, as a consequence of the results in Table I, (a). In regime (I), the highest and second highest VBs have vertical masses $m_{1}$ and $m_{2}$, respectively, with $m_{1}<m_{2}$. This case is illustrated schematically in Fig. 8(I). If a well is wide and quantum confinement is weak, the order of $E_{1, n=1}$ and $E_{2, n=1}$ should be the same as in the strained film case in Eq. (8). However, if $L_{\mathrm{w}}$ decreases, the subband energy with lighter mass is confined much more compared to the heavier mass. Consequently, the order of subband energies is reversed, at approximately $2 \mathrm{~nm}$, and we find $E_{1, n=1}<E_{2, n=1}$ in a very narrow QW. Actually, $\Delta E$ of $L_{\mathrm{w}}=\infty$ is very large, so that instead of energy and optical polarization reversing, we find

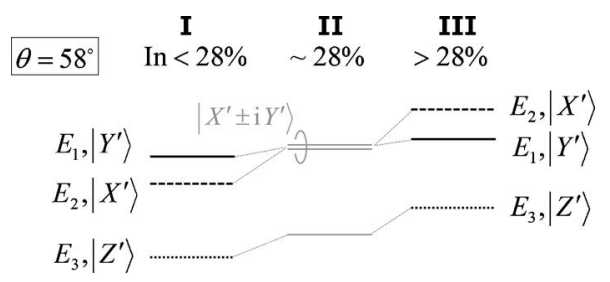

FIG. 6. The VB energy diagram dependence on In content for semipolar InGaN/GaN systems. they simply decrease, as shown in Figs. 7(a) and 7(b). Nonpolar InGaN/GaN with any In content is assigned to this regime, as mentioned before.

\section{Regime II: $E_{1} \sim E_{2}$}

The lines with In content $x$ of $25 \%, 28 \%$, and $30 \%$ correspond to regime (II). In this regime, the topmost two VB eigenenergies become close and band-mixing is significant even for wide wells. Thus, the absolute values of optical polarization are small. Furthermore, VB masses are also close to $m_{\mathrm{HH}}$ and $m_{\mathrm{LH}}$, as determined earlier. Their values along the $z^{\prime}$-axis are nearly constant, as indicated in Table I, (b), so that $|\Delta E|$ is virtually insensitive to them in the case of $x=25 \%$ or slightly sensitive for $x=28 \%$. At $x=30 \%$, the overall trend for $|\Delta E|$ as a function of $L_{\mathrm{w}}$ is similar to that of regime (III), however, $|\Delta E|$ is much smaller.

\section{Regime III: $E_{1}<E_{2}$}

$\mathrm{In}_{0.4} \mathrm{Ga}_{0.6} \mathrm{~N} / \mathrm{GaN}$ belongs to regime (III). In this case, $|\Delta E|$ is insensitive to wider well width as in regime (I). Despite this, $|\Delta E|$ constantly increases for thinner wells due to the relations $m_{1}<m_{2}$ and $E_{2}<E_{1}$, as schematically drawn in Fig. 8. 
(a)

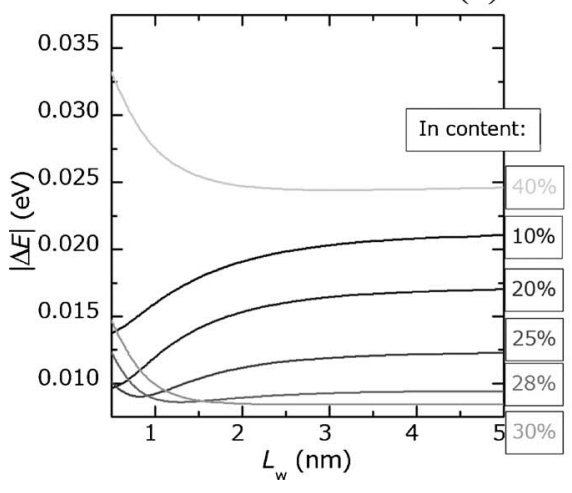

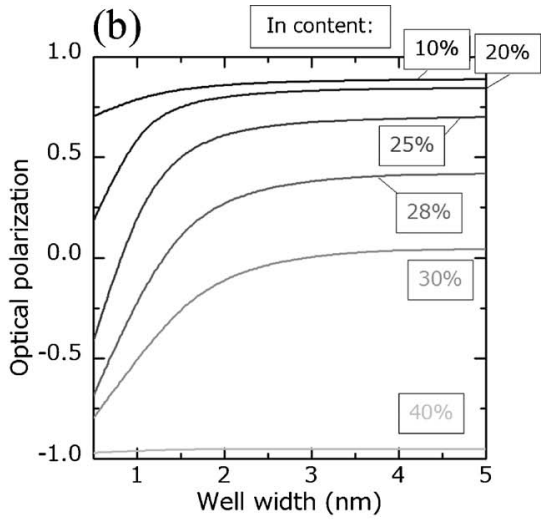

FIG. 7. (a) $|\Delta E|$ vs $L_{\mathrm{w}}$, and (b) optical polarization vs $L_{\mathrm{w}}$ calculated for a $(11 \overline{2} 2) \mathrm{InGaN} / \mathrm{GaN}$ QW with In content $x$.

\section{CONCLUSIONS}

In summary, we have investigated theoretically the VB mass characteristics of non- $c$-plane nitride semiconductor heterostructures, both in strained films and QWs. Analytical expressions for VB masses are given for the cases of $E_{1}$ $>E_{2}$ and $E_{1}<E_{2}$. We find that both the VB mass and overall trends become similar to those of the $c$-plane case when $E_{1}$ $\sim E_{2}$. We show that the in-plane VB mass is reduced to $7.3 \%$ along a certain direction and $\sim 26.9 \%$ with in-plane averaging for the nonpolar and semipolar cases with $E_{1}>E_{2}$ or $E_{1}<E_{2}$. Moreover, due to less coupling of the topmost two VBs, VB mass becomes nearly independent of $k$, so that the analytical VB mass reproduces band structure with high reliability by a simple parabolic approximation. Furthermore,
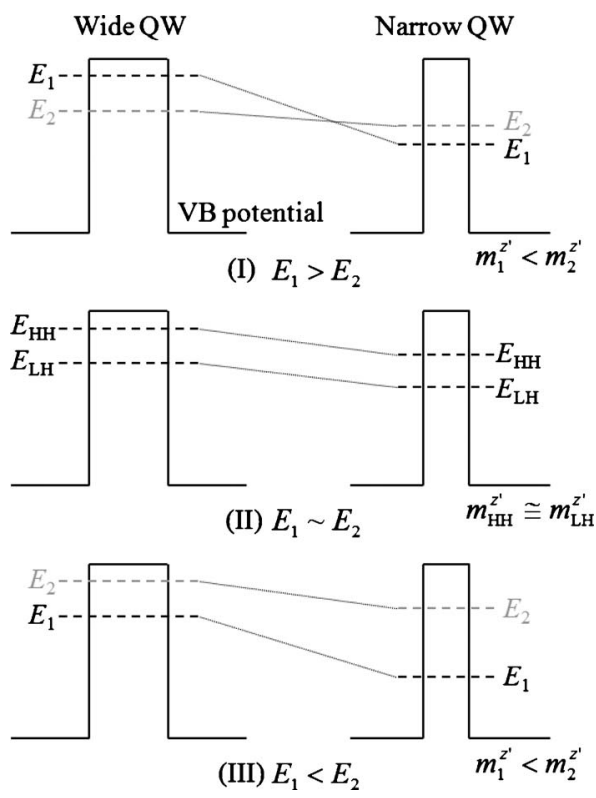

FIG. 8. A schematic of subband energy diagrams in QWs in regimes (I), (II), and (III), where only the first subbands are depicted and subband index $n=1$ is omitted for simplicity. for the semipolar case with $E_{1} \sim E_{2}$, we show that the $c$-plane approximation is valid for band calculations. We also find that vertical VB mass is always heavy in any crystal orientation. However, there is a small difference between the topmost two VBs, so that the formation of subbands in QWs has different features among the regimes (I), (II), and (III), depending on the order of VB eigenenergies.

${ }^{1}$ T. Takeuchi, S. Sota, M. Katsuragawa, M. Komori, H. Takeuchi, H. Amano, and I. Akasaki, Jpn. J. Appl. Phys., Part 2 36, L382 (1997).

${ }^{2}$ A. E. Romanov, T. J. Baker, S. Nakamura, and J. S. Speck, J. Appl. Phys. 100, 023522 (2006).

${ }^{3}$ M. Funato, M. Ueda, Y. Kawakami, Y. Narukawa, T. Kosugi, M. Takahashi, and T. Mukai, Jpn. J. Appl. Phys., Part 2 45, L659 (2006).

${ }^{4}$ Y. Enya, Y. Yoshizumi, T. Kyono, K. Akita, M. Ueno, M. Adachi, T. Sumitomo, S. Tokuyama, T. Ikegami, K. Katayama, and T. Nakamura, Appl. Phys. Express 2, 082101 (2009).

${ }^{5}$ K. Kojima, M. Funato, Y. Kawakami, S. Masui, S. Nagahama, and T. Mukai, Appl. Phys. Lett. 91, 251107 (2007).

${ }^{6}$ S. Ghosh, P. Waltereit, O. Brandt, H. T. Grahn, and K. H. Ploog, Phys. Rev. B 65, 075202 (2002).

${ }^{7}$ M. Ueda, M. Funato, K. Kojima, Y. Kawakami, Y. Narukawa, and T. Mukai, Phys. Rev. B 78, 233303 (2008).

${ }^{8}$ M. Feneberg, F. Lipski, R. Sauer, K. Thonke, P. Brückner, B. Neubert, T. Wunderer, and F. Scholz, J. Appl. Phys. 101, 053530 (2007).

${ }^{9}$ A. A. Yamaguchi, Jpn. J. Appl. Phys., Part 2 46, L789 (2007).

${ }^{10}$ K. Kojima, H. Kamon, M. Funato, and Y. Kawakami, Phys. Status Solidi C 5, 3038 (2008).

${ }^{11}$ K. Kojima, M. Funato, Y. Kawakami, S. Nagahama, and T. Mukai, Abstract of the International Workshop on Nitride semiconductors, Montreux, Switzerland, 2008.

${ }^{12}$ T. Ohtoshi, A. Niwa, and T. Kuroda, Jpn. J. Appl. Phys., Part 2 35, L1566 (1996).

${ }^{13}$ S. H. Park and S. L. Chuang, Phys. Rev. B 59, 4725 (1999).

${ }^{14}$ E. O. Kane, J. Phys. Chem. 1, 249 (1957).

${ }^{15}$ G. L. Bir and G. E. Pikus, Symmetry and Strain-Induced Effects in Semiconductors (Wiley, NewYork, 1974).

${ }^{16}$ T. Uenoyama and M. Suzuki, Appl. Phys. Lett. 67, 2527 (1995).

${ }^{17}$ S. L. Chuang and C. S. Chang, Phys. Rev. B 54, 2491 (1996).

${ }^{18}$ K. Kojima, H. Kamon, M. Funato, and Y. Kawakami, Jpn. J. Appl. Phys. 48, 080201 (2009).

${ }^{19}$ R. Winkler and U. Rössler, Phys. Rev. B 488918 (1993).

${ }^{20}$ S. L. Chuang and C. S. Chang, Semicond. Sci. Technol. 12, 252 (1997).

${ }^{21}$ K. Kim, W. Lambrecht, B. Segall, and M. Schilfgaarde, Phys. Rev. B 56, 7363 (1997)

${ }^{22}$ I. Vurgaftman and J. R. Meyer, J. Appl. Phys. 94, 3675 (2003). 